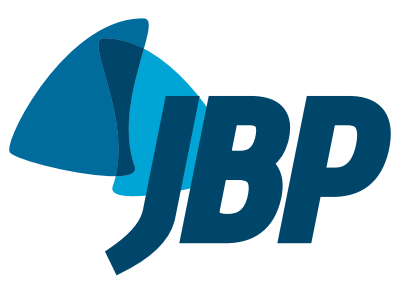

1. Universidade Católica de Pelotas, Pelotas (RS) Brasil.

2. Programa de Pós-Graduação em Epidemiologia, Universidade Federal de Pelotas, Pelotas (RS) Brasil.

a. (iD) http://orcid.org/0000-0003-3935-5207

b. (iD) http://orcid.org/0000-0002-4640-2254

c. (iD http://orcid.org/0000-0001-7137-1747

Submitted: 28 May 2018

Accepted: 23 September 2018

Study carried out in the Programa de Pós-Graduação em Epidemiologia,

Universidade Federal de Pelotas, Pelotas (RS), Brazil.

\section{Temporal evolution of and factors associated with asthma and wheezing in schoolchildren in Brazil}

\author{
Gabriela Ávila Marques ${ }^{1,2, a}$, Andrea Wendt²,b, Fernando César Wehrmeister ${ }^{2, c}$
}

\begin{abstract}
Objective: To estimate the evolution of the prevalence of asthma and wheezing among schoolchildren in Brazil from 2012 to 2015, as well as to identify factors associated with both conditions. Methods: This was a cross-sectional study using data from the Brazilian National School-Based Adolescent Health Survey for 2012 and 2015. To characterize the evolution of the prevalence of asthma and wheezing, we used linear regression with weighted-least-squares estimation and presented the annual percent change (APC). Results: During the study period, there was a reduction in the prevalence of wheezing, from $23.2 \%$ in 2012 to $22.4 \%$ in 2015 (APC, -0.27 ). The prevalence of asthma increased from $12.4 \%$ in 2012 to $16.0 \%$ in 2015 (APC, 1.20). The increase in the prevalence of asthma was greatest in the southern region of the country (APC, 2.17). Having any history of smoking and having consumed alcohol in the last 30 days were factors that influenced the prevalence of wheezing and the prevalence of a self-reported diagnosis of asthma during the two years evaluated. Conclusions: There has been an increase in the prevalence of asthma in recent years in Brazil. Our data underscore the importance of improving health strategies and policies aimed the control of asthma.
\end{abstract}

Keywords: Asthma/epidemiology; Respiratory sounds; Students.

\section{INTRODUCTION}

Asthma is considered a chronic noncommunicable disease (CNCD) whose symptoms appear early in life in approximately half of the cases. ${ }^{(1)}$ Its most common symptom is wheezing, which is caused by bronchospasms and airway obstruction. (2) Although episodes of cough, chest tightness, and wheezing, among others, are important for characterizing the condition, there are no rules as to whether or not these symptoms must be present for it to be considered asthma, which represents a diagnostic challenge. (3) The International Study of Asthma and Allergies in Childhood (ISAAC) reports a mean prevalence of asthma symptoms of $14.1 \%$ in adolescents aged 13 to 14 years worldwide, whereas in Latin American countries the rates are above $20 \%$. $^{(4)}$

In 1988, the prevalence of asthma in Brazil was already among the highest in the world, ${ }^{(5,6)}$ and, apparently, these estimates continue to rise. The prevalence of asthma in children and adolescents evaluated between 1998 and 2008 increased from 8.6\% (1998) to 9.1\% (2008) for those living in urban areas, and from $4.9 \%$ to $5.9 \%$ for those living in rural areas. ${ }^{(7)}$ Therefore, it is important to identify the associated factors to gain a better understanding of the disease. These factors are related to environmental, social, and demographic conditions, as well as to lifestyle. ${ }^{(8)}$

Asthma can also have a negative impact on quality of life, because it results in direct and indirect costs to the population in the form of medical appointments, use of medication, hospitalizations, loss of productivity, work/school absenteeism, and early mortality. ${ }^{(9-13)}$ In Brazil, there was a $36 \%$ decrease in the number of hospitalizations between 2008 and 2013; the northern, northeastern, and southeastern regions are the ones with the highest hospitalization rates in the country. In that same period, the cost of hospitalizations reached nearly USD 170 million. (14) A systematic review found that the cost of hospitalizations and medications for the treatment of asthma is of USD 733 per person per year. ${ }^{(15)}$

Considering this scenario, monitoring asthma and its risk factors is key for the development of enhanced health strategies and policies, especially because the condition can be treated at the primary level of health care. ${ }^{(16)}$ Therefore, the objective of the present study was to estimate the prevalence of asthma and wheezing in schoolchildren in the years of 2012 and 2015, and to verify the factors associated with them.

\section{METHODS}

This was a cross-sectional study using data from the 2012 and 2015 editions of the Pesquisa Nacional de Saúde do Escolar (PeNSE, Brazilian National School-Based Adolescent Health Survey). The PeNSE is a school-based survey conducted by the Brazilian Ministry of Health in partnership with the Brazilian Institute of Geography and Statistics, whose purpose is to collect information on adolescent health. The survey covers various health-related aspects (socioeconomic condition, family context, eating

Correspondence to:

Gabriela Ávila Marques. Centro de Pesquisas Epidemiológicas, Universidade Federal de Pelotas, Rua Marechal Deodoro, 1160, $3^{\circ}$ piso, Caixa Postal 464, Centro CEP 96020-220, Pelotas, RS, Brazil.

Tel./Fax: 5553 3284-1300. E-mail: gabriamarques@gmail.com

Financial support: None. 
habits, physical activity level etc.). To measure such aspects, we used straightforward self-administered questionnaires that adolescents can easily understand.

The fist edition of the PeNSE happened in 2009 (with schoolchildren from Brazilian state capitals only), followed by the second and third editions in 2012 and 2015 , respectively. We chose to use data from these last two surveys because the sample was expanded to other municipalities in addition to the state capitals. ${ }^{(17,18)}$ The 2012 and 2015 editions of the PeNSE surveyed middle school students from public and private institutions located in urban and rural areas throughout Brazil. The PeNSE target population was formed by Year 9 students (former $8^{\text {th }}$ grade) because adolescents of this age group already have reasonable autonomy to complete the self-administered questionnaire. The student sample was composed of children attending schools in the 26 state capitals, the Federal District, and other selected municipalities. ${ }^{(17,18)}$

Our study used the occurrence of asthma in life and wheezing in the previous 12 months as outcomes, measured through the following questions: a) "Have you ever had asthma in your life?" (yes/no); and b) "Have you had any wheezing (squeaking) in your chest in the last 12 months?" (yes/no). The independent variables were as follows: gender (female/male); age ( $\leq 13$ years, 14 years, or $\geq 15$ years); skin color (white, black, brown, yellow, or indigenous); maternal educational attainment (no schooling, complete primary education, incomplete primary education, complete secondary education, or higher education or above). The following questions were used to measure adolescent and parental smoking, respectively: "Have you ever smoked cigarettes, even if only one or two drags?" (yes/no), and "Does any of your parents or guardians smoke?" (none of them, my father or male guardian only, my mother or female guardian only, both my parents or guardians, or I do not know). Alcohol consumption in the previous 30 days was measured by the question: "In the last 30 days, on how many days did you drink at least one glass or dose of alcohol?". For the present study, we considered the consumption of at least one dose of alcohol in the previous 30 days (yes/no). We also calculated an economic index using a principal component analysis based on the ownership of the following goods/services: landline, cell phone, computer, Internet, car, motorcycle, and number of bathrooms with a shower in the household. We also took into consideration whether or not participants had household help. Then, the index was divided into quintiles, the first quintile corresponded to the poorest economic index.

As for the statistical analysis, first, we described the sample of the two editions of the survey, and then we presented the prevalence of asthma and wheezing, as well as their respective $95 \%$ CIs. We also presented gross and outcome-adjusted analyses for asthma and wheezing in the two years evaluated, using the Poisson regression and the respective $95 \%$ CIs to estimate prevalence ratios (PR). For the outcome-adjusted analyses, the associations between independent variables and outcomes were adjusted for each variable.

To evaluate the evolution of asthma and wheezing in 2012 and 2015, we used a linear regression with weighted-least-squares estimation, and then we presented the annual percent change (APC) for each outcome in each Brazilian region and countrywide. All analyses were conducted with Stata statistical package, version 12.1 (StataCorp LP, College Station, TX, USA), and command svy, which considers the study design.

The PeNSE was approved by the Research Ethics Board of the Brazilian Ministry of Health under report number 192/2012 (CONEP/MS Registry number 16805 of March 27, 2012). Student participation was voluntary; students were informed they were free not to participate, or not to complete parts of or the entire questionnaire, should they wish to do so. All student and school details were collected and kept confidential.

\section{RESULTS}

The student samples in the 2012 and 2015 editions of the survey were composed of 109,104 and 102,072 individuals, respectively. The 2012 and 2015 sample characteristics are shown in Tables 1 and 2. In both editions, most of the students were female $(52.2 \%$ in 2012 and $51.3 \%$ in 2015), were 14 years of age (45.6\% and $51.0 \%$ in 2012 and 2015, respectively), and had brown skin color (42.2\% in 2012 and $43.1 \%$ in 2015). Also in both editions, approximately $20 \%$ of the participants reported having some history of smoking and $25 \%$ reported having consumed alcohol in the previous 30 days.

Figure 1 shows wheezing prevalence rates in 2012 and 2015. In Brazil, wheezing decreased from 23.2\% to $22.4 \%$ in 2015 (APC: -0.27 ). Considering the evolution of wheezing in each Brazilian region, we see that only the northern and center-western regions presented significant reductions, with an APC of -0.47 and -0.43 , respectively.

Figure 2 shows the evolution of asthma (reported in life) in both editions (2012 and 2015). In Brazil as a whole, there was an increase from $12.4 \%$ to $16.0 \%$ in the prevalence of asthma (APC: 1.2 ). The centerwestern region was the only one that did not present a significant change in the prevalence of asthma. All other regions showed significant increases, the greatest one in the southern region (APC: 2.17).

Table 3 shows the prevalence ratios of factors associated with wheezing in 2012 and in 2015. After adjustments, in 2012, the following factors showed a positive association with a higher prevalence of wheezing: being a girl (PR $=1.16,95 \% \mathrm{CI}: 1.12-1.21)$, having a higher economic index $(P R=1.20 ; 95 \% \mathrm{CI}$ : 1.11-1.29), having any history of smoking ( $P R=1.39$, 95\% CI: 1.33-1.45), and having consumed alcohol in the previous 30 days ( $P R=1.28,95 \% \mathrm{CI}: 1.33-1.45$ ). Age was also associated, as older adolescents presented a lower prevalence of wheezing. In 2015, the factors associated with a higher prevalence of wheezing were 
Table 1. Description of the 2012 and 2015 samples of the Brazilian National School-Based Adolescent Health Survey.

\begin{tabular}{|c|c|c|}
\hline Variables & $\begin{array}{l}2012 \\
\text { n (\%) }\end{array}$ & $\begin{array}{l}2015 \\
\text { n (\%) }\end{array}$ \\
\hline \multicolumn{3}{|l|}{ Gender } \\
\hline Male & $52,015(47.8)$ & $49,290(48.7)$ \\
\hline Female & $57,089(52.2)$ & $52,782(51.3)$ \\
\hline \multicolumn{3}{|l|}{ Age, years } \\
\hline$\leq 13$ & $22,443(22.9)$ & $17,260(18.3)$ \\
\hline 14 & $50,900(45.6)$ & $51,611(51.0)$ \\
\hline$\geq 15$ & $35,761(31.6)$ & $33,201(30.7)$ \\
\hline \multicolumn{3}{|l|}{ Skin color } \\
\hline White & $37,674(36.8)$ & $33,775(36.2)$ \\
\hline Black & $14,513(13.4)$ & $12,849(13.4)$ \\
\hline Brown & $48,237(42.2)$ & $46,935(43.1)$ \\
\hline Yellow/Indigenous & $8,611(7.6)$ & $8,405(7.4)$ \\
\hline \multicolumn{3}{|l|}{ Maternal educational attainment } \\
\hline No schooling & $7,371(10.1)$ & $5,531(7.4)$ \\
\hline Complete primary education & $25,951(32.2)$ & $18,217(26.5)$ \\
\hline Incomplete primary education & $156,975(18.1)$ & $12,299(17.1)$ \\
\hline Complete secondary education & $28,244(28.8)$ & 23,359 (30.9) \\
\hline Higher education & $13,036(10.9)$ & $17,232(18.1)$ \\
\hline \multicolumn{3}{|l|}{ Economic index } \\
\hline 1st quintile & $21,725(21.4)$ & $22,634(20.7)$ \\
\hline 2nd quintile & $23,821(22.2)$ & $19,320(18.1)$ \\
\hline 3rd quintile & $22,738(21.2)$ & $22,383(22.6)$ \\
\hline 4th quintile & $32,056(29.7)$ & $29,022(32.4)$ \\
\hline 5 th quintile & $8,249(5.6)$ & $8,239(6.3)$ \\
\hline \multicolumn{3}{|l|}{ Any history of smoking } \\
\hline No & $86,113(80.4)$ & $83,158(81.6)$ \\
\hline Yes & $22,784(19.6)$ & $18,723(18.4)$ \\
\hline \multicolumn{3}{|l|}{ Alcohol previous 30 days } \\
\hline No & 80,905 (73.9) & $79,364(76.2)$ \\
\hline Yes & $27,763(26.1)$ & $22,597(23.8)$ \\
\hline \multicolumn{3}{|l|}{ Parents who smoke } \\
\hline None & $76,809(70.2)$ & $75,098(73.2)$ \\
\hline One parent only & $24,138(24.2)$ & $20,562(22.3)$ \\
\hline Both & $5,022(5.6)$ & $3,800(4.5)$ \\
\hline TOTAL & $109,704(100)$ & $102,072(100)$ \\
\hline
\end{tabular}

similar to those of 2012; however, having one parent/ guardian who smokes (PR $=1.15,95 \% \mathrm{CI}: 1.09-1.21$ ), or both $(P R=1.24,95 \% \mathrm{CI}: 1.13-1.37)$ were also associated this time.

Table 4 describes the prevalence of factors associated with asthma in 2012 and in 2015. After adjustments, in 2012, the following factors were associated with asthma: having yellow or indigenous skin color (PR = 1.16; 95\% CI: 1.05-1.28), having a higher maternal educational attainment ( $P R=1.25 ; 95 \% \mathrm{CI}: 1.11-1.40)$, having a higher economic index $(P R=1.27 ; 95 \% \mathrm{CI}$ : 1.14-1.42), having any history of smoking ( $P R=1.38$; $95 \%$ CI: $1.30-1.47)$, and having consumed alcohol in the previous 30 days ( $P R=1.23,95 \% \mathrm{CI}: 1.16-1.30$ ). In 2015, the factors associated remained the same, except for skin color, which was not associated with the outcome this time, and having parents/guardians who smoke, which was (PR $=1.16,95 \% \mathrm{CI}: 1.00-1.35)$.

\section{DISCUSSION}

The results of the present study indicate a decrease in the prevalence of wheezing from $23.2 \%$ in 2012 to $22.4 \%$ in 2015 . The data related to asthma, in turn, indicate an increase in the prevalence of this CNCD from 2012 to 2015 (APC: 1.2). The main factors associated with wheezing in 2012 were gender, age, and economic level. Having smoked at least once in life and having consumed alcohol in the previous 30 days also influenced the prevalence of wheezing and the self-reported diagnosis of asthma in both years.

One of the possible explanations for the increase in the prevalence of asthma and a concomitant decrease in wheezing is the different reporting periods for one or another. Also, wheezing is a symptom that is used as a proxy for the disease in epidemiological studies, especially in the period of life between childhood and adolescence, and, unlike asthma, it does not require 
Table 2. Distribution of the prevalence of wheezing and asthma in the Brazilian National School-Based Adolescent Health Survey, 2012 and 2015.

\begin{tabular}{|c|c|c|c|c|}
\hline \multirow[t]{2}{*}{ Variables } & \multicolumn{2}{|c|}{2012} & \multicolumn{2}{|c|}{2015} \\
\hline & $\begin{array}{l}\text { Wheezing } \\
\%(95 \% \mathrm{Cl})\end{array}$ & $\begin{array}{c}\text { Asthma } \\
\%(95 \% \mathrm{Cl})\end{array}$ & $\begin{array}{l}\text { Wheezing } \\
\% \text { (95\% CI) }\end{array}$ & $\begin{array}{c}\text { Asthma } \\
\%(95 \% \mathrm{CI})\end{array}$ \\
\hline \multicolumn{5}{|l|}{ Gender } \\
\hline Male & $21.4(20,8-22,0)$ & $12.8(12.4-13.3)$ & $19.2(18.5-19.8)$ & $15.6(15.0-16.1)$ \\
\hline Female & $24.9(24.3-25.4)$ & $12.1(11.7-12.5)$ & $25.4(24.7-26.1)$ & $16.5(15.9-17.0)$ \\
\hline \multicolumn{5}{|l|}{ Age, years } \\
\hline$\leq 13$ & $23.6(22.7-24.5)$ & $11.7(11.1-12.4)$ & $24.0(22.7-25.3)$ & $16.7(15.6-17.8)$ \\
\hline 14 & $23.4(22.8-24.0)$ & $12.3(11.9-12.7)$ & $22.5(21.8-23.1)$ & $15.7(15.1-16.2)$ \\
\hline$\geq 15$ & $22.6(21.9-23.3)$ & $13.1(12.6-13.7)$ & $21.2(20.4-22.0)$ & $16.2(15.5-16.9)$ \\
\hline \multicolumn{5}{|l|}{ Skin color } \\
\hline White & $23.3(22.7-24.0)$ & $12.6(12.1-13.2)$ & $22.3(21.5-23.1)$ & $16.1(15.4-16.8)$ \\
\hline Black & $22.7(21.6-23.8)$ & $12.0(11.1-12.8)$ & $20.5(19.2-21.7)$ & $16.0(14.9-17.1)$ \\
\hline Brown & $23.1(22.5-23.7)$ & $12.0(11.6-12.5)$ & $22.7(22.0-23.4)$ & $15.7(15.1-16.3)$ \\
\hline Yellow/Indigenous & $24.2(22.7-25.6)$ & $14.2(13.1-15.4)$ & $24.2(22.5-25.9)$ & $17.8(16.3-19.3)$ \\
\hline \multicolumn{5}{|l|}{ Maternal educational attainment } \\
\hline No schooling & $22.7(21.3-24.1)$ & $12.6(11.5-13.6)$ & $21.3(19.5-23.0)$ & $14.6(13.0-16.1)$ \\
\hline Complete primary education & $23.1(22.3-23.9)$ & $11.8(11.2-12.3)$ & $23.1(22.0-24.2)$ & $15.7(14.8-16.7)$ \\
\hline Incomplete primary education & $23.8(22.8-24.9)$ & $12.3(11.6-13.1)$ & $23.7(22.3-25.1)$ & $16.4(15.2-17.6)$ \\
\hline Complete secondary education & $23.9(23.0-24.7)$ & $13.5(12.9-14.1)$ & $24.3(23.2-25.3)$ & $17.3(16.4-18.1)$ \\
\hline Higher education & $26.3(25.0-27.7)$ & $16.6(15.6-17.8)$ & $23.2(22.0-24.4)$ & $19.1(18.0-20.3)$ \\
\hline \multicolumn{5}{|l|}{ Economic index } \\
\hline 1st quintile & $20.9(20.1-21.7)$ & $11.1(10.5-11.7)$ & $19.7(18.8-20.6)$ & $13.6(12.9-14.4)$ \\
\hline 2nd quintile & $22.6(21.8-23.5)$ & $12.3(11.7-12.9)$ & $22.2(21.1-23.2)$ & $16.3(15.4-17.2)$ \\
\hline 3rd quintile & $23.2(22.3-24.1)$ & $11.8(11.1-12.4)$ & $22.6(21.6-23.6)$ & $16.2(15.3-17.0)$ \\
\hline 4th quintile & $25.0(24.2-25.8)$ & $13.1(12.5-13.7)$ & $24.0(23.1-25.0)$ & $16.4(15.7-17.2)$ \\
\hline 5th quintile & $25.6(24.0-27.2)$ & $17.1(15.7-18.4)$ & $22.7(20.9-24.4)$ & $20.3(18.5-22.0)$ \\
\hline \multicolumn{5}{|l|}{ Any history of smoking } \\
\hline No & $21.1(20.7-21.6)$ & $11.4(11.1-11.7)$ & $20.6(20.1-21.1)$ & $15.0(14.5-15.4)$ \\
\hline Yes & $31.7(30.7-32.7)$ & $16.7(15.9-17.4)$ & $30.3(29.0-31.6)$ & $20.8(19.8-21.9)$ \\
\hline \multicolumn{5}{|l|}{ Alcohol previous 30 days } \\
\hline No & $20.9(20.5-21.4)$ & $11.4(11.0-11.7)$ & $20.4(19.8-20.9)$ & $14.9(14.5-15.4)$ \\
\hline Yes & $29.7(28.9-30.5)$ & $15.4(14.8-16.1)$ & $28.8(27.8-29.9)$ & $19.6(18.7-20.5)$ \\
\hline \multicolumn{5}{|l|}{ Parents who smoke } \\
\hline None & $22.5(22.0-22.9)$ & $12.2(11.8-12.5)$ & $21.1(20.5-21.6)$ & $15.5(15.0-16.0)$ \\
\hline One parent only & $24.8(23.9-25.7)$ & $12.6(12.0-13.2)$ & $25.2(24.1-26.3)$ & $17.0(16.1-17.9)$ \\
\hline Both & $24.8(23.0-26.7)$ & $13.8(12.4-15.2)$ & $28.3(25.7-31.0)$ & $14.5(16.2-20.8)$ \\
\hline TOTAL & $23.2(22.8-23.6)$ & $12.4(12.1-12.7)$ & $22.4(21.9-22.8)$ & $16.0(15.6-16.4)$ \\
\hline
\end{tabular}

a physician to be diagnosed. The increase we found in the prevalence of asthma can be related to an improved access to health care, especially at the primary level, observed in Brazil. ${ }^{(19-21)}$ On the other hand, the decrease in wheezing in 2015 can be related to a better control of the disease, resulting from the asthma medication provided for free via the Popular Pharmacy Program the previous year. ${ }^{(22)}$

There is evidence of an increasing prevalence of asthma from 1960 onwards, but its causes have yet to be fully understood. (23) A study published in 2009 using ISAAC data showed a high prevalence of asthma in Latin America and stated that it was associated with social and biological mechanisms related to economically unfavorable conditions and urbanization, as well as to exposure to allergens, irritants, and pollutants. ${ }^{(20)}$ In the present study, we found a positive association between a higher economic level and a higher prevalence of wheezing and asthma, which can be explained by a more favorable family financial condition that facilitates access to health care and diagnosis. In addition, a higher maternal educational attainment can mean more access to information about the disease and, as a consequence, a higher quality of reporting by this group of participants. ${ }^{(24)}$ A lower maternal educational attainment is associated with lower incomes and limited access to health care, which leads to lack of medication and undertreatment. $(10,25,26)$

Having yellow or indigenous skin color has also shown to be a factor associated with asthma in the 2012 edition of the survey. As a consequence of discrimination, ethnic minorities have poorer access to 


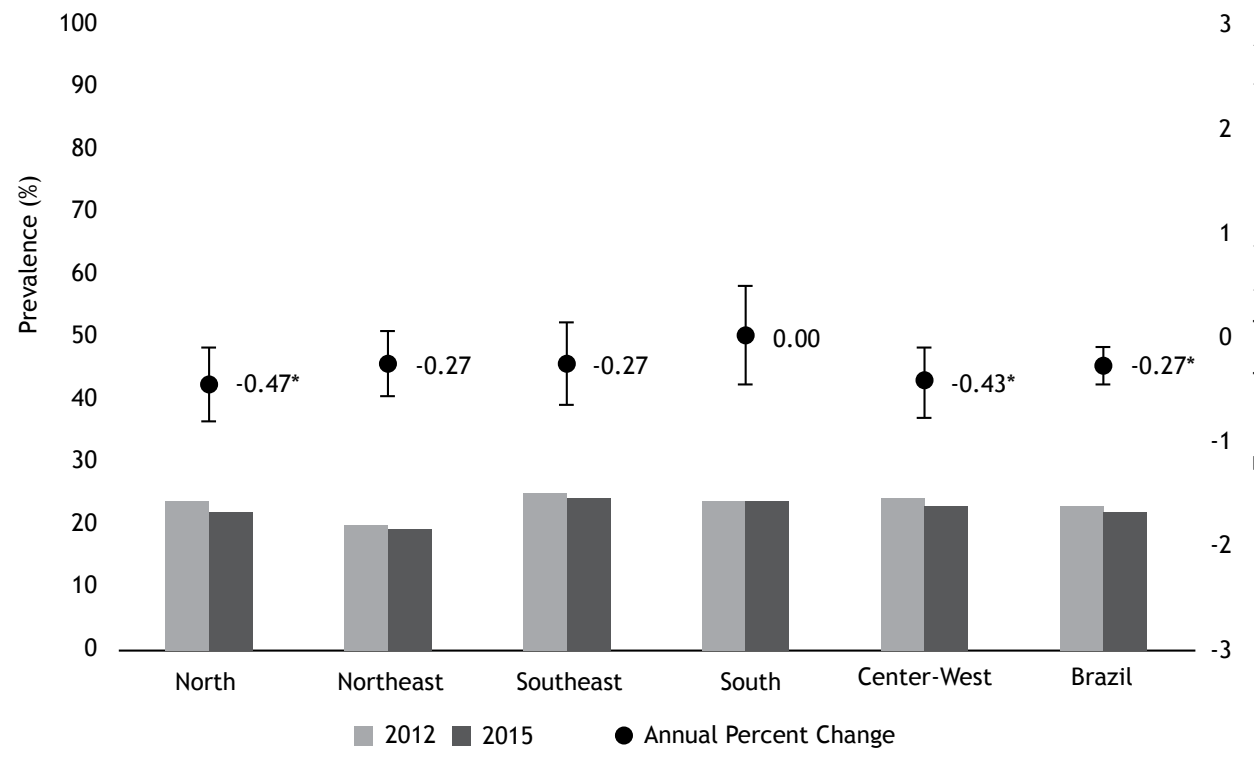

Figure 1. Prevalence of wheezing in 2012 and 2015 per Brazilian region.

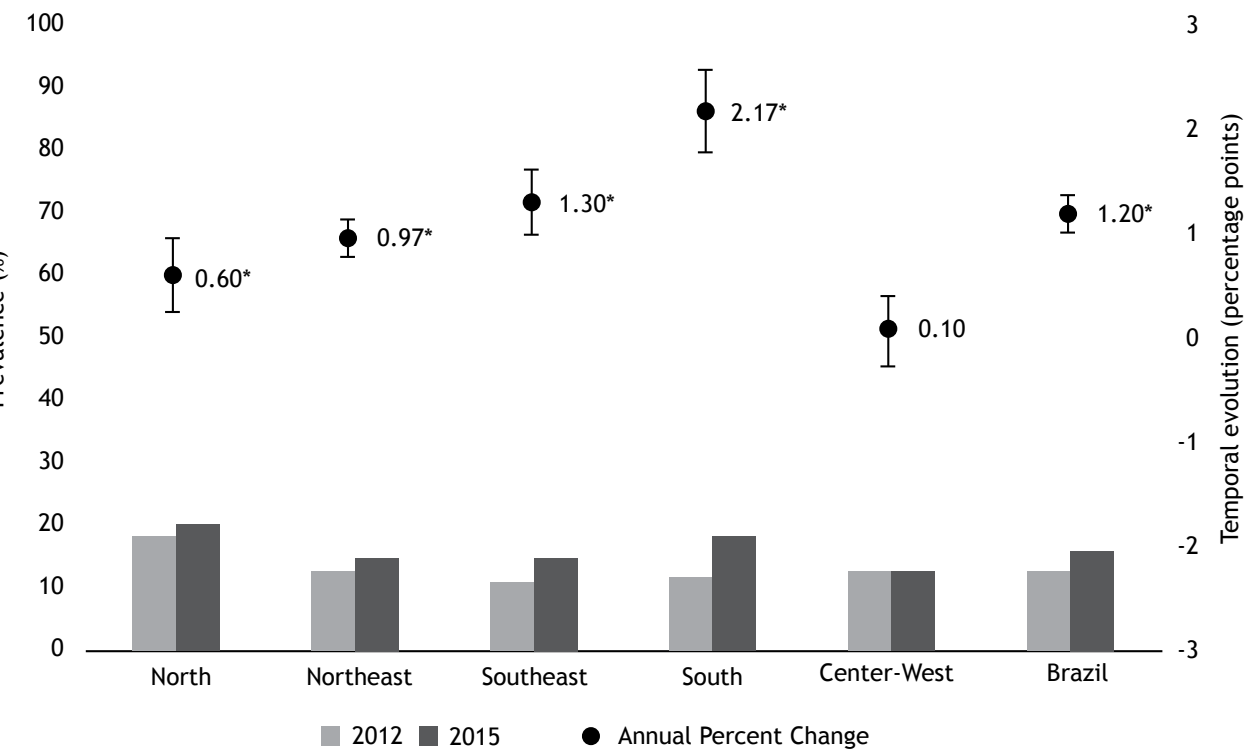

Figure 2. Prevalence of asthma in 2012 and 2015 per Brazilian region.

goods, opportunities, and health care. Therefore, people who are in a situation of socioeconomic marginalization, living in terrible housing conditions in the outskirts of Brazilian cities, are more susceptible to diseases in general, including asthma. $(27,28)$

As for the biological mechanisms, wheezing was more prevalent in female and younger participants. A group of authors ${ }^{(7)}$ who studied children between zero and 9 years of age found a higher rate of asthma diagnosis in boys, possibly due to physiological disadvantages presented by boys in this age group, such as lower respiratory rates and a higher concentration of $\mathrm{IgE}$ (allergen-specific antibodies). $(7,29)$ One of the possible explanations for this is that the risk of boys developing wheezing and asthma reduces in late childhood and, therefore, in adolescence, the disease becomes more prevalent in girls. ${ }^{(30)}$

Exposure to allergens, irritants, and pollutants might be associated with an increased prevalence of asthma. (2) In the present study, having smoked and having consumed alcohol were also associated with the prevalence of asthma in 2012 and 2015, as well as having one or both parents/guardians who smoked. A study with Hispanic children living in the southwest of the USA found a positive association between exposure to cigarette smoke in places with limited air circulation and asthma. (31) According to the Brazilian guidelines for asthma management, ${ }^{(32)}$ smoking hinders asthma control, whereas quitting smoking improves the quality of life of individuals and decreases the asthma costs 
Table 3. Gross and adjusted prevalence ratios of factors associated with wheezing. Brazilian National School-Based Adolescent Health Survey, 2012 and 2015.

\begin{tabular}{|c|c|c|c|c|c|c|c|c|}
\hline \multirow[t]{2}{*}{ Variables } & \multicolumn{4}{|c|}{2012} & \multicolumn{4}{|c|}{2015} \\
\hline & Gross & $\mathbf{p}$ & Adjusted & $\mathbf{p}$ & Gross & p & Adjusted & $\mathbf{p}$ \\
\hline Gender & & $<0.001$ & & $<0.001$ & & $<0.001$ & & $<0.001$ \\
\hline Male & - & & - & & - & & - & \\
\hline Female & $\begin{array}{c}1.16 \\
(1.12-1.20)\end{array}$ & & $\begin{array}{c}1.16 \\
(1.12-1.21)\end{array}$ & & $\begin{array}{c}1.32 \\
(1.27-1.38)\end{array}$ & & $\begin{array}{c}1.31 \\
(1.25-1.37)\end{array}$ & \\
\hline Age, years & & 0.130 & & 0.001 & & 0.001 & & $<0.001$ \\
\hline$\leq 13$ & - & & - & & - & & - & \\
\hline 14 & $\begin{array}{c}0.99 \\
(0.95-1.04)\end{array}$ & & $\begin{array}{c}0.97 \\
(0.93-1.02)\end{array}$ & & $\begin{array}{c}0.94 \\
(0.88-0.99)\end{array}$ & & $\begin{array}{c}0.92 \\
(0.86-0.98)\end{array}$ & \\
\hline$\geq 15$ & $\begin{array}{c}0.96 \\
(0.91-1.01)\end{array}$ & & $\begin{array}{c}0.92 \\
(0.87-0.96)\end{array}$ & & $\begin{array}{c}0.88 \\
(0.83-0.94)\end{array}$ & & $\begin{array}{c}0.85 \\
(0.79-0.91)\end{array}$ & \\
\hline Skin color & & 0.410 & & 0.371 & & 0.003 & & 0.021 \\
\hline White & - & & - & & - & & - & \\
\hline Black & $\begin{array}{c}0.97 \\
(0.92-0.13)\end{array}$ & & $\begin{array}{c}1.00 \\
(0.94-1.06)\end{array}$ & & $\begin{array}{c}0.92 \\
(0.85-0.98)\end{array}$ & & $\begin{array}{c}0.95 \\
(0.89-1.03)\end{array}$ & \\
\hline Brown & $\begin{array}{c}0.99 \\
(0.95-1.03)\end{array}$ & & $\begin{array}{c}1.01 \\
(0.97-1.06)\end{array}$ & & $\begin{array}{c}1.01 \\
(0.97-1.07)\end{array}$ & & $\begin{array}{c}1.04 \\
(0.99-1.09)\end{array}$ & \\
\hline Yellow/Indigenous & $\begin{array}{c}1.04 \\
(0.97-1.11)\end{array}$ & & $\begin{array}{c}1.06 \\
(1.00-1.14)\end{array}$ & & $\begin{array}{c}1.08 \\
(1.00-1.17)\end{array}$ & & $\begin{array}{c}1.08 \\
(1.00-1.17)\end{array}$ & \\
\hline Maternal educational attainment & & $<0.001$ & & 0.107 & & 0.077 & & 0.532 \\
\hline No schooling & - & & - & & - & & - & \\
\hline Complete primary education & $\begin{array}{c}1.02 \\
(0.95-1.09)\end{array}$ & & $\begin{array}{c}0.99 \\
(0.92-1.06)\end{array}$ & & $\begin{array}{c}1.08 \\
(0.98-1.19)\end{array}$ & & $\begin{array}{c}1.02 \\
(0.93-1.13)\end{array}$ & \\
\hline Incomplete primary education & $\begin{array}{c}1.05 \\
(0.97-1.13)\end{array}$ & & $\begin{array}{c}1.01 \\
(0.94-1.10)\end{array}$ & & $\begin{array}{c}1.11 \\
(1.01-1.23)\end{array}$ & & $\begin{array}{c}1.04 \\
(0.94-1.15)\end{array}$ & \\
\hline Complete secondary education & $\begin{array}{c}1.05 \\
(0.98-1.13)\end{array}$ & & $\begin{array}{c}1.00 \\
(0.93-1.08)\end{array}$ & & $\begin{array}{c}1.14 \\
(1.04-1.25)\end{array}$ & & $\begin{array}{c}1.06 \\
(0.96-1.17)\end{array}$ & \\
\hline Higher education & $\begin{array}{c}1.16 \\
(1.07-1.26)\end{array}$ & & $\begin{array}{c}1.08 \\
(0.99-1.18)\end{array}$ & & $\begin{array}{c}1.09 \\
(0.99-1.20)\end{array}$ & & $\begin{array}{c}1.01 \\
(0.91-1.13)\end{array}$ & \\
\hline Economic index & & $<0.001$ & & $<0.001$ & & $<0.001$ & & $<0.001$ \\
\hline 1st quintile & - & & - & & - & & - & \\
\hline 2nd quintile & $\begin{array}{c}1.08 \\
(1.03-1.14)\end{array}$ & & $\begin{array}{c}1.07 \\
(1.01-1.13)\end{array}$ & & $\begin{array}{c}1.13 \\
(1.06-1.20)\end{array}$ & & $\begin{array}{c}1.12 \\
(1.05-1.19)\end{array}$ & \\
\hline 3rd quintile & $\begin{array}{c}1.11 \\
(1.05-1.17)\end{array}$ & & $\begin{array}{c}1.10 \\
(1.04-1.16)\end{array}$ & & $\begin{array}{c}1.15 \\
(1.08-1.22)\end{array}$ & & $\begin{array}{c}1.15 \\
(1.08-1.22)\end{array}$ & \\
\hline 4th quintile & $\begin{array}{c}1.20 \\
(1.14-1.26)\end{array}$ & & $\begin{array}{c}1.18 \\
(1.12-1.24)\end{array}$ & & $\begin{array}{c}1.22 \\
(1.15-1.30)\end{array}$ & & $\begin{array}{c}1.22 \\
(1.15-1.30)\end{array}$ & \\
\hline 5th quintile & $\begin{array}{c}1.23 \\
(1.14-1.32)\end{array}$ & & $\begin{array}{c}1.20 \\
(1.11-1.29)\end{array}$ & & $\begin{array}{c}1.15 \\
(1.05-1.26)\end{array}$ & & $\begin{array}{c}1.17 \\
(1.06-1.29)\end{array}$ & \\
\hline Any history of smoking & & $<0.001$ & & $<0.001$ & & $<0.001$ & & $<0.001$ \\
\hline No & - & & - & & - & & - & \\
\hline Yes & $\begin{array}{c}1.50 \\
(1.44-1.56)\end{array}$ & & $\begin{array}{c}1.39 \\
(1.33-1.45)\end{array}$ & & $\begin{array}{c}1.47 \\
(1.40-1.54)\end{array}$ & & $\begin{array}{c}1.35 \\
(1.28-1.42)\end{array}$ & \\
\hline Alcohol previous 30 days & & $<0.001$ & & $<0.001$ & & $<0.001$ & & $<0.001$ \\
\hline No & - & & - & & - & & - & \\
\hline Yes & $\begin{array}{c}1.42 \\
(1.37-1.47)\end{array}$ & & $\begin{array}{c}1.28 \\
(1.33-1.45)\end{array}$ & & $\begin{array}{c}1.42 \\
(1.35-1.48)\end{array}$ & & $\begin{array}{c}1.25 \\
(1.19-1.32)\end{array}$ & \\
\hline Parents who smoke & & $<0.001$ & & 0.05 & & $<0.001$ & & $<0.001$ \\
\hline None & - & & - & & - & & - & \\
\hline One parent only & $\begin{array}{c}1.10 \\
(1.06-1.15)\end{array}$ & & $\begin{array}{c}1.06 \\
(1.01-1.10)\end{array}$ & & $\begin{array}{c}1.20 \\
(1.14-1.26)\end{array}$ & & $\begin{array}{c}1.15 \\
(1.09-1.21)\end{array}$ & \\
\hline Both & $\begin{array}{c}1.11 \\
(1.02-1.19)\end{array}$ & & $\begin{array}{c}1.04 \\
(0.96-1.13)\end{array}$ & & $\begin{array}{c}1.34 \\
(1.22-1.48)\end{array}$ & & $\begin{array}{c}1.24 \\
(1.13-1.37)\end{array}$ & \\
\hline
\end{tabular}

to the health care system. Not only has tobacco a negative impact on the management of asthma, exacerbating its symptoms, but cocaine, heroin, and alcohol do as well. 
Table 4. Gross and adjusted prevalence ratios of factors associated with asthma. Brazilian National School-Based Adolescent Health Survey, 2012 and 2015.

\begin{tabular}{|c|c|c|c|c|c|c|c|c|}
\hline \multirow[t]{2}{*}{ Variables } & \multicolumn{4}{|c|}{2012} & \multicolumn{4}{|c|}{2015} \\
\hline & Gross & p & Adjusted & $\mathbf{p}$ & Gross & $\mathbf{p}$ & Adjusted & $\mathbf{p}$ \\
\hline Gender & & 0.012 & & 0.273 & & 0.032 & & 0.004 \\
\hline Male & - & & - & & - & & - & \\
\hline Female & $\begin{array}{c}0.94 \\
(0.90-0.99)\end{array}$ & & $\begin{array}{c}0.97 \\
(90.92-1.02)\end{array}$ & & $\begin{array}{c}1.56 \\
(1.00-1.11)\end{array}$ & & $\begin{array}{c}1.09 \\
(1.03-1.15)\end{array}$ & \\
\hline Age, years & & 0.003 & & 0.174 & & 0.219 & & 0.203 \\
\hline$\leq 13$ & - & & - & & - & & - & \\
\hline 14 & $\begin{array}{c}1.05 \\
(0.98-1.12)\end{array}$ & & $\begin{array}{c}1.01 \\
(0.94-1.08)\end{array}$ & & $\begin{array}{c}0.94 \\
(0.87-1.01)\end{array}$ & & $\begin{array}{c}0.94 \\
(0.86-1.02)\end{array}$ & \\
\hline$\geq 15$ & $\begin{array}{c}1.12 \\
(1.05-1.20)\end{array}$ & & $\begin{array}{c}1.06 \\
(0.99-1.15)\end{array}$ & & $\begin{array}{c}0.97 \\
(0.90-1.05)\end{array}$ & & $\begin{array}{c}0.97 \\
(0.89-1.07)\end{array}$ & \\
\hline Skin color & & 0.002 & & 0.024 & & 0.069 & & 0.141 \\
\hline White & - & & - & & - & & - & \\
\hline Black & $\begin{array}{c}0.95 \\
(0.88-1.03)\end{array}$ & & $\begin{array}{c}1.01 \\
(0.93-1.10)\end{array}$ & & $\begin{array}{c}0.99 \\
(0.92-1.08)\end{array}$ & & $\begin{array}{c}1.03 \\
(0.94-1.14)\end{array}$ & \\
\hline Brown & $\begin{array}{c}0.95 \\
(0.90-1.01)\end{array}$ & & $\begin{array}{c}1.02 \\
(0.96-1.08)\end{array}$ & & $\begin{array}{c}0.98 \\
(0.92-1.04)\end{array}$ & & $\begin{array}{c}1.02 \\
(0.96-1.09)\end{array}$ & \\
\hline Yellow/Indigenous & $\begin{array}{c}1.12 \\
(1.03-1.23)\end{array}$ & & $\begin{array}{c}1.16 \\
(1.05-1.28)\end{array}$ & & $\begin{array}{c}1.11 \\
(1.01-1.22)\end{array}$ & & $\begin{array}{c}1.14 \\
(1.02-1.27)\end{array}$ & \\
\hline Maternal educational attainment & & $<0.001$ & & $<0.001$ & & $<0.001$ & & 0.002 \\
\hline No schooling & - & & - & & - & & - & \\
\hline Complete primary education & $\begin{array}{c}0.94 \\
(0.85-1.03)\end{array}$ & & $\begin{array}{c}0.92 \\
(0.84-1.02)\end{array}$ & & $\begin{array}{c}1.08 \\
(0.96-1.22)\end{array}$ & & $\begin{array}{c}1.02 \\
(0.90-1.16)\end{array}$ & \\
\hline Incomplete primary education & $\begin{array}{c}0.98 \\
(0.88-1.09)\end{array}$ & & $\begin{array}{c}0.97 \\
(0.87-1.08)\end{array}$ & & $\begin{array}{c}1.13 \\
(0.99-1.28)\end{array}$ & & $\begin{array}{c}1.06 \\
(0.93-1.21)\end{array}$ & \\
\hline Complete secondary education & $\begin{array}{c}1.07 \\
(0.98-1.18)\end{array}$ & & $\begin{array}{c}1.05 \\
(0.94-1.16)\end{array}$ & & $\begin{array}{c}1.18 \\
(1.05-1.33)\end{array}$ & & $\begin{array}{c}1.11 \\
(0.98-1.26)\end{array}$ & \\
\hline Higher education & $\begin{array}{c}1.32 \\
(1.19-1.47)\end{array}$ & & $\begin{array}{c}1.25 \\
(1.11-1.40)\end{array}$ & & $\begin{array}{c}1.31 \\
(1.17-1.48)\end{array}$ & & $\begin{array}{c}1.23 \\
(1.07-1.40)\end{array}$ & \\
\hline Economic index & & $<0.001$ & & $<0.001$ & & $<0.001$ & & $<0.001$ \\
\hline 1st quintile & - & & - & & - & & - & \\
\hline 2nd quintile & $\begin{array}{c}1.11 \\
(1.03-1.19)\end{array}$ & & $\begin{array}{c}1.08 \\
(1.00-1.17)\end{array}$ & & $\begin{array}{c}1.19 \\
(1.10-1.29)\end{array}$ & & $\begin{array}{c}1.22 \\
(1.11-1.34)\end{array}$ & \\
\hline 3rd quintile & $\begin{array}{c}1.06 \\
(0.98-1.15)\end{array}$ & & $\begin{array}{c}1.03 \\
(0.95-1.12)\end{array}$ & & $\begin{array}{c}1.19 \\
(1.10-1.28)\end{array}$ & & $\begin{array}{c}1.20 \\
(1.10-1.31)\end{array}$ & \\
\hline 4th quintile & $\begin{array}{c}1.18 \\
(1.10-1.26)\end{array}$ & & $\begin{array}{c}1.09 \\
(1.01-1.19)\end{array}$ & & $\begin{array}{c}1.20 \\
(1.12-1.29)\end{array}$ & & $\begin{array}{c}1.16 \\
(1.06-1.27)\end{array}$ & \\
\hline 5th quintile & $\begin{array}{c}1.54 \\
(1.40-1.69)\end{array}$ & & $\begin{array}{c}1.27 \\
(1.14-1.42)\end{array}$ & & $\begin{array}{c}1.49 \\
(1.34-1.64)\end{array}$ & & $\begin{array}{c}1.36 \\
(1.20-1.53)\end{array}$ & \\
\hline Any history of smoking & & $<0.001$ & & $<0.001$ & & $<0.001$ & & $<0.001$ \\
\hline No & - & & - & & - & & - & \\
\hline Yes & $\begin{array}{c}1.46 \\
(1.38-1.54)\end{array}$ & & $\begin{array}{c}1.38 \\
(1.30-1.47)\end{array}$ & & $\begin{array}{c}1.39 \\
(1.31-1.48)\end{array}$ & & $\begin{array}{c}1.25 \\
(1.16-1.35)\end{array}$ & \\
\hline Alcohol previous 30 days & & $<0.001$ & & $<0.001$ & & $<0.001$ & & $<0.001$ \\
\hline No & - & & - & & - & & - & \\
\hline Yes & $\begin{array}{c}1.36 \\
(1.29-1.43)\end{array}$ & & $\begin{array}{c}1.23 \\
(1.16-1.30)\end{array}$ & & $\begin{array}{c}1.31 \\
(1.24-1.39)\end{array}$ & & $\begin{array}{c}1.17 \\
(1.09-1.25)\end{array}$ & \\
\hline Parents who smoke & & 0.045 & & 0.360 & & 0.001 & & 0.042 \\
\hline None & - & & - & & - & & - & \\
\hline One parent only & $\begin{array}{c}1.03 \\
(0.98-1.10)\end{array}$ & & $\begin{array}{c}0.99 \\
(0,93-1.06)\end{array}$ & & $\begin{array}{c}1.10 \\
(1.03-1.17)\end{array}$ & & $\begin{array}{c}1.07 \\
(0.99-1.15)\end{array}$ & \\
\hline Both & $\begin{array}{c}1.14 \\
(1.02-1.26)\end{array}$ & & $\begin{array}{c}1.08 \\
(0,96-1.22)\end{array}$ & & $\begin{array}{c}1.19 \\
(1.05-1.35)\end{array}$ & & $\begin{array}{c}1.16 \\
(1.00-1.35)\end{array}$ & \\
\hline
\end{tabular}

Our data show that there was a reduction in the prevalence of wheezing in Brazil, especially in the northern region (APC: -0.27 ); however, the prevalence of asthma increased in the same region despite the fact 
that there was greater coverage of public health care assistance programs and of the Family Health Strategy initiative in 2015 than in 2012. ${ }^{(21)}$ Another study found a higher prevalence of a medical diagnosis of asthma in the north of Brazil (13.5\%; 95\% CI: $12.7-14.2)$ than in the other regions. ${ }^{(24)}$ The prevalence of wheezing also decreased in the center-western region, whereas the prevalence of asthma did not present a statistically significant difference. Other authors ${ }^{(21)}$ reported an increase in the prevalence rate from $21.6 \%$ in 1998 to $30.1 \%$ in 2013 ( 8.5 percentage points) based on data from visits that took place via private health care plans in that region. This could be understood as a higher quality of treatment provided by the private health care network, though there was also an increase in the participation of the Unified Health System (Brazilian public health care system).

In addition to the northern region, the northeastern, southeastern, and southern regions also presented increases in the prevalence of asthma in life. According to the American Thoracic Society, climate and temperature can be considered irritants that trigger asthma. ${ }^{(33)}$ For asthma patients, places with a wide temperature variation or high levels of pollution can be harmful. ${ }^{(34,35)}$ A study ${ }^{(36)}$ concluded that climate changes can alter local climate patterns-such as maximum and minimum temperatures, and precipitation from rain and storms-and have negative impacts on allergic diseases, which could explain the increased prevalence of asthma in climatically distinct regions. In addition, according to a study with data from throughout Brazil, (37) lower temperatures, such as those found in the south of the country, tend to keep people indoors for longer periods of time, with reduced air circulation, which leads to an increase in allergic processes and respiratory infections. A positive association was also found between the prevalence of active asthma and water deprivation in the northeastern region. ${ }^{(38)}$

Controlling risk factors-such as smoking, alcohol consumption, and exposure to allergens-is considered important for a better management and treatment of asthma. ${ }^{(39)}$ Therefore, despite the increase in the prevalence of asthma, the decrease in the prevalence of its main symptom suggests a reasonably positive management by the health care system.
We should point out that our study has a few limitations. One of them is the different reporting periods for asthma (in life) and wheezing (in the previous 12 months). Another one is related to the fact that the PeNSE is a school-based survey conducted throughout the country, focusing on several aspects of adolescent health. As such, the establishment of a medical diagnosis or the inclusion of further questions to better define asthma for the entire study population might have been logistically infeasible and certain pieces of information might not have been collected. In addition, the fact that the questionnaire is selfadministered may lead to errors since participants might misunderstand some of the questions or make mistakes when choosing the answers. These limitations can prevent this study from being compared with others that focus on respiratory diseases, such as the ISAAC, which describes not only the prevalence but also the severity of asthma, rhinitis, and eczema, and contains more information on risk factors for these diseases. ${ }^{(4,5)}$ However, among the strengths of the our study are the representative sample of schoolchildren from throughout the country and the continuity in asthma monitoring, which can contribute to improving disease control strategies. Despite their limitations, self-reported questions have been used in population studies as a good proxy for the monitoring of wheezing and, consequently, asthma.

Our results suggest a reduction in the prevalence of wheezing but an increase in the prevalence of asthma in the years evaluated. Social and biological mechanisms, such as an economically unfavorable condition, limited access to health care (and consequently poorer diagnoses), lack of medication and treatment, as well as urbanization, climate, and temperature, can lead to a higher prevalence of the disease. A higher prevalence of asthma, in turn, has negative effects on quality of life and increases health costs with medical appointments, use of medication, frequency of hospitalization, loss of productivity, work/school absenteeism, in addition to early mortality. Therefore, a better understanding of the factors associated with the increased prevalence of asthma is essential for the development of health strategies and policies.

\section{REFERENCES}

1. Barreto ML, Ribeiro-Silva Rde C, Malta DC, Oliveira-Campos M Andreazzi MA, Cruz AA. Prevalence of asthma symptoms among adolescents in Brazil: National Adolescent School-based Health Survey (PeNSE 2012). Rev Bras Epidemiol. 2014;17 Suppl 1:106-15 https://doi.org/10.1590/1809-4503201400050009

2. Martinez JA. Not all that wheezes is asthma! J Bras Pneumol. 2013;39(4):518-20. https://doi.org/10.1590/S180637132013000400017

3. Sociedade Brasileira de Pneumologia e Tisiologia. Diretrizes da Sociedade Brasileira de Pneumologia e Tisiologia para o manejo da asma - 2012. J Bras Pneumol. 2012;38(Suppl 1):S1-S46.

4. Lai CK, Beasley R, Crane J, Foliaki S, Shah J, Weiland S; et al. Global variation in the prevalence and severity of asthma symptoms: phase three of the International Study of Asthma and Allergies in Childhood (ISAAC). Thorax. 2009;64(6):476-83. https://doi.org/10.1136/ thx.2008.106609

5. Worldwide variations in the prevalence of asthma symptoms: the International Study of Asthma and Allergies in Childhood (ISAAC) Eur Respir J. 1998;12(2):315-35. https://doi.org/10.1183/09031936.9 8.12020315

6. Worldwide variation in prevalence of symptoms of asthma allergic rhinoconjunctivitis, and atopic eczema: ISAAC. The International Study of Asthma and Allergies in Childhood (ISAAC) Steering Committee. Lancet. 1998;351(9111):1225-32. https://doi. org/10.1016/S0140-6736(97)07302-9

7. Wehrmeister FC, Menezes AM, Cascaes AM, Martínez-Mesa J, Barros AJ. Time trend of asthma in children and adolescents in Brazil, 1998-2008. Rev Saude Publica. 2012;46(2):242-50. https://doi. org/10.1590/S0034-89102012005000008

8. Muiño A, Menezes AM, Reichert FF, Duquia RP, Chatkin M Wheezing phenotypes from birth to adolescence: a cohort study in Pelotas, Brazil, 1993-2004 [Article in Portuguese]. J Bras 
Pneumol. 2008;34(6):347-55. https://doi.org/10.1590/S180637132008000600003

9. Terzano C, Cremonesi G, Girbino G, Ingrassia E, Marsico S, Nicolini $G$, et al. 1-year prospective real life monitoring of asthma control and quality of life in Italy. Respir Res. 2012;13:112. https://doi. org/10.1186/1465-9921-13-112

10. Braman SS. The global burden of asthma. Chest. 2006;130(1 Suppl):4S-12S. https://doi.org/10.1378/chest.130.1_suppl.4S

11. Pitrez PM, Stein RT. Asthma in Latin America: the dawn of a new epidemic. Curr Opin Allergy Clin Immunol. 2008;8(5):378-83. https:// doi.org/10.1097/ACl.0b013e32830fb911

12. Brasilia Itália C. S. Ache; Kahan B, Fiterman J. Prevalence of asthma symptoms and treatment of children and adolescents from 2 to 14 years of age in Porto Alegre, Rio Grande do Sul, Brazil. J Bras Pneumol. 2005;31(2):103-10. http://dx.doi.org/10.1590/S180637132005000200004

13. Araújo DV, Mesquita ET. Economia da saúde: ferramenta para tomada de decisão em medicina. Rev SOCERJ. 2003;16(4):258-61.

14. Cardoso TA, Roncada C, Silva ERD, Pinto LA, Jones MH, Stein RT, et al. The impact of asthma in Brazil: a longitudinal analysis of data from a Brazilian national database system. J Bras Pneumol. 2017;43(3):163168. https://doi.org/10.1590/s1806-37562016000000352

15. Stirbulov R, Lopes da Silva N, Maia SC, Carvalho-Netto E, Angelini L. Cost of severe asthma in Brazil-systematic review. J Asthma. 2016;53(10):1063-70. https://doi.org/10.3109/02770903.2016.1171 338

16. Nedel FB, Facchini LA, Martín-Mateo M, Vieira LAS, Thumé E. Family Health Program and ambulatory care-sensitive conditions in Southern Brazil. Rev Saude Publica. 2008;42(6):1041-52. https://doi. org/10.1590/S0034-89102008000600010

17. Penna G. Pesquisa Nacional da Saúde do Escolar (PeNSE). Cienc Saude Colet. 2012;15(Suppl 2):3006. https://doi.org/10.1590/S141381232010000800001

18. Brasil. Instituto Brasileiro de Geografia e Pesquisa (IBGE) [homepage on the Internet]. Rio de Janeiro: IBGE; [updated 2016; cited 2018 Sep 1]: Pesquisa Nacional de Saúde do Escolar (PeNSE) 2015. [Adobe Acrobat document, 131p.]. Available from: https://biblioteca.ibge. gov.br/visualizacao/livros/liv97870.pdf

19. Facchini LA, Piccini RX, Tomasi E, Thumé E, Silveira DS, Siqueira FV, et al. Performance of the PSF in the Brazilian South and Northeast: institutional and epidemiological Assessment of Primary Health Care [Article in Portuguese]. Cienc Saude Colet. 2006;11(3):669-81. https://doi.org/10.1590/S1413-81232006000300015

20. Cooper PJ, Rodrigues LC, Cruz AA, Barreto ML. Asthma in Latin America : a public heath challenge and research opportunity. Allergy. 2009;64(1):5-17. https://doi.org/10.1111/j.1398-9995.2008.01902.x

21. Viacava $F$, Bellido JG. Health, access to services and sources of payment, according to household surveys Cien Saude

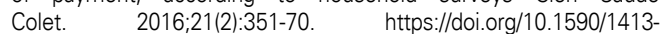
81232015212.19422015

22. da Silva RM, Caetano R. "Farmácia Popular do Brasil" Program: characterization and evolution between 2004 and 2012. Cienc Saude Colet. 2015:20(10):2943-56. https://doi.org/10.1590/1413812320152010.17352014

23. Beasley R, Crane J, Lai CK, Pearce N. Epidemiology and genetics of asthma Prevalence and etiology of asthma. 2000;105(2):S466-S472. https://doi.org/10.1016/S0091-6749(00)90044-7

24. Kuschnir FC, Gurgel RQ, Solé D, Costa E, Felix MM, de Oliveira CL, et al. ERICA: Prevalence of asthma in Brazilian adolescents. Rev
Saude Publica. 2016;50 Suppl 1:13s. https://doi.org/10.1590/s015188787.2016050006682

25. Chong Neto HJ, Rosário NA, Solé D; Latin American ISAAC Group. Asthma and Rhinitis in South America: How Different They are From Other Parts of the World. Allergy Asthma Immunol Res. 2012;4(2):62 7. https://doi.org/10.4168/aair.2012.4.2.62

26. Mallol J, Solé D, Baeza-Bacab M, Aguirre-Camposano V, Soto-Quiros

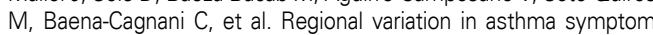
prevalence in Latin American children. J Asthma. 2010;47(6):644-50. https://doi.org/10.3109/02770901003686480

27. Coimbra CE Jr, Santos RV. Health, minorities and inequality: some webs of inter-relations, emphasizing indigenous peoples in Brazi [Article in Portuguese]. Cien Saude Colet. 2000;5(1):125-32. https:// doi.org/10.1590/S1413-81232000000100011

28. Chor D, Lima CR. Epidemiologic aspects of racial inequalities in health in Brazil [Article in Portuguese]. Cad Saude Publica. 2005;21(5):158694. https://doi.org/10.1590/S0102-311X2005000500033

29. Sears MR, Burrows B, Flannery EM, Herbison GP, Holdaway MD. Atopy in childhood. I. Gender and allergen related risks for development of hay fever and asthma. Clin Exp Allergy. 1993;23(11):941-8. $\quad$ https://doi.org/10.1111/j.1365-2222.1993. tb00279.x

30. Fuhlbrigge $A L$, Jackson $B$, Wright RJ. Gender and asthma. Immunol Allergy Clin N Am. 2002;22(4):753-89. https://doi.org/10.1016/S0889$8561(02) 00022-X$

31. Gonzales M, Malcoe LH, Myers OB, Espinoza J. Risk factors for asthma and cough among Hispanic children in the southwestern United States of America, 2003-2004. Rev Panam Salud Publica. 2007;21(5):274-81. https://doi.org/10.1590/S102049892007000400002

32. Sociedade Brasileira de Pneumologia e Tisiologia. IV Diretrizes Brasileiras para o Manejo da Asma. J Bras Pneumol. 2006;32(Suppl 7):S447-S474. http://dx.doi.org/10.1590/S1806-37132006001100002 https://doi.org/10.1590/S1806-37132006001100002

33. Maslan J, Mims JW. What is asthma? Pathophysiology, demographics, and health care costs. Otolaryngol Clin North Am. 2014;47(1):13-22. https://doi.org/10.1016/j.otc.2013.09.010

34. Evans SE, Scanlon PD. Current practice in pulmonary function testing. Mayo Clin Proc. 2003;78(6):758-63; quiz 763. https://doi. org/10.4065/78.6.758

35. Taketomi EA, Marra SMG, Segundo GRS. Fisioterapia em asma: efeito na função pulmonar e em parâmetros imunológicos. Fit Perform J. 2005;4(2):97-100. https://doi.org/10.3900/fpj.4.2.97.p

36. D'Amato GD, Vitale C, Rosario N, Neto HJC, Chong-Silva DC, Mendonça $F$, et al. Climate change, allergy and asthma, and the role of tropical forests. World Allergy Organ J. 2017;10(1):11. https://doi. org/10.1186/s40413-017-0142-7

37. Wehrmeister $F C$, Peres KG Regional inequalities in the prevalence of asthma diagnosis in children: an analysis of the Brazilian National Household Sample Survey, 2003 [Article in Portuguese]. Cad Saude Publica. 2010;26(9):1839-52. http://dx.doi.org/10.1590/S0102 311X2010000900017

38. Franco JM, Gurgel R, Sole D, Lúcia França V, Brabin B; Brazilian ISAAC Group. Socio-environmental conditions and geographical variability of asthma prevalence in Northeast Brazil. Allergol Immunopathol. 2009;37(3):116-21. https://doi.org/10.1016/S0301-0546(09)71722-7

39. Chatkin MN, Menezes AM. Prevalence and risk factors for asthma in schoolchildren in southern Brazil. J Pediatr (Rio J). 2005;81(5):411-6. https://doi.org/10.2223/JPED.1393 